\title{
Effects of pharmaceutical care on adherence and persistence to bisphosphonates in postmenopausal osteoporotic women
}

\author{
P. S. M. Lai ${ }^{*}$ PhD, S. S. Chua $\dagger$ PhD, Y. Y. Chew BSc and S. P. Chan§ MBBS FRCP (Edin) \\ ${ }^{*}$ Medical Education Research and Development Unit, Faculty of Medicine, University of Malaya, Kuala \\ Lumpur, †Department of Pharmacy, Faculty of Medicine, University of Malaya, Kuala Lumpur, \$Central \\ Diagnostic Laboratory, University Malaya Medical Centre, Kuala Lumpur, and §Department of Medicine, \\ Faculty of Medicine, University of Malaya, Kuala Lumpur, Malaysia
}

\section{SUMMARY}

What is known and Objective: Studies have shown that comprehensive interventions by pharmacists can improve adherence and persistence to osteoporosis therapy, but the association between adherence and bone turnover markers (BTMs) has never been studied. Therefore, the aim of this study was to evaluate the effects of pharmaceutical care on medication adherence (and its effects on BTMs), as well as persistence of postmenopausal osteoporotic women to prescribed bisphosphonates.

Methods: A randomized controlled trial was conducted from 2005 to 2009 in the University Malaya Medical Centre, Malaysia. Inclusion criteria: postmenopausal osteoporotic women diagnosed with osteoporosis with a $T$-score $\leq-2.5$ or who had a low-trauma fracture and prescribed weekly alendronate/risedronate. Intervention participants received counselling on osteoporosis, risk factors, lifestyle modifications, goals of therapy, side effects and the importance of adherence. Adherence was assessed at months 3, 6 and 12, and persistence at month 12. Feedback on BTMs was provided at months 4 and 7. The control group received no counselling. Two BTMs were used: serum C-terminal cross-linking telopeptide of type I collagen (CTX-I) and serum osteocalcin (OC). Main outcomes measured: medication adherence, BTMs and persistence.

Received 06 April 2010, Accepted 12 July 2010

Correspondence: Dr P. S. M. Lai, Medical Education Research and Development Unit, Faculty of Medicine, University of Malaya, 50603 Kuala Lumpur, Malaysia. Tel.: 60379675739 ; fax: 603796 75760; e-mail: plai@ummc.edu.my
Results and Discussion: Intervention participants who received pharmaceutical care reported significantly higher medication adherence at 6 $(P=0.015)$ and 12 months $(P=0.047)$ compared with the control group; but this effect was not shown by the BTMs. This is probably due to the long effect of bisphosphonates in bone. A significant difference was found between serum CTX-I and OC in identifying nonresponders to anti-resorptive therapy $(P<0 \cdot 001)$, indicating the usefulness of BTMs as an objective marker. However, pharmaceutical care did not affect persistence to osteoporosis therapy within a 1-year period [log rank (Mantel-Cox) $\left.\chi^{2}=0.496, P=0 \cdot 481\right]$. The proportion of participants who were persistent with bisphosphonate therapy after 12 months was $89 \cdot 8 \%$ and $87.0 \%$ in the control and intervention group respectively.

What is new and Conclusion: The provision of pharmaceutical care improved medication adherence but not persistence. BTMs were not appropriate objective measures for assessing adherence to weekly bisphosphonates but were useful for identifying non-responders to treatment within 3-6 months, much earlier than using bone mineral density. The study indicates that pharmacists have a role in improving medication adherence, but its long-term effect on persistence warrants further studies with longer duration.

Keywords: adherence, bone turnover markers, osteoporosis, persistence, pharmaceutical care, postmenopausal women 


\section{WHAT IS KNOWN AND OBJECTIVE}

The ultimate goal of management in osteoporosis is to prevent fracture, and currently, bisphosphonates are the drugs of choice (1). However, bisphosphonates need to be taken in a specific manner and for at least a year for evidence of improvement of bone mineral density (BMD) and protection against fracture to emerge $(2,3)$. Approximately $50-75 \%$ of women, prescribed anti-osteoporosis drug therapy, no longer take them 12 months after initiation of treatment (4). Poor adherence leads to lower therapeutic efficacy $(5,6)$, weaker suppression of bone resorption (7), smaller increases in BMD (8), less reduction in fracture risk (9) and drug wastage (6).

By using a multi-faceted approach, physicians, dietitians, physiotherapists and nurses can successfully improve the outcomes of patients with osteoporosis by monitoring medication adherence and persistence (10). A systematic review conducted to assess the effect of such non-drug interventions on community-dwelling postmenopausal osteoporotic women (11) found three studies that assessed medication adherence (12-14) and persistence (12, $13,15)$ respectively. It was concluded that such interventions were better in improving adherence than in improving persistence $(66 \cdot 7 \%$ vs. $33 \cdot 3 \%)$.

Pharmacists are strategically in an excellent position to provide pharmaceutical care, which is defined as the responsible provision of drug therapy for the purpose of achieving definite outcomes that improve quality of life (QOL) (16). Previous prospective, observational studies showed that comprehensive interventions by pharmacists improved adherence to osteoporosis therapy, but this effect has not been objectively measured using bone turnover markers (BTMs) (17-19). BTMs, which reflect the rates of bone turnover, can predict the risk of osteoporosis-related fractures (20), assess adherence and effectiveness of osteoporosis therapy, and detect treatment failure (nonresponders) (21). BTMs are markedly reduced within 3-6 months of anti-resorptive therapy (22). If necessary, changes in treatment can be made as soon as possible, saving almost 2 years of inappropriate therapy, as reliable BMD results can only be obtained after 2 years.

There is little published evidence from randomized controlled study of the effects of involvement of pharmacists in the management of osteoporosis. Therefore, the primary objective of this study was to evaluate the effects of pharmaceutical care on medication adherence and its possible association with BTMs, as well as persistence of postmenopausal osteoporotic women to their prescribed bisphosphonates using this study design.

\section{METHODS}

This randomized controlled study was conducted from September 2005 to February 2009 at the University Malaya Medical Centre (UMMC) to assess adherence (and its effect on BTMs), persistence, QOL, knowledge and patient satisfaction of postmenopausal osteoporosis patients. Results on QOL, knowledge and patient satisfaction will be reported elsewhere. Approval from the Medical Ethics Committee, UMMC was obtained before commencement of the study.

\section{Patients}

Inclusion criteria. Postmenopausal women who had just been diagnosed with osteoporosis (BMD $T$-score $\leq-2 \cdot 5 /$ low-trauma fracture sustained at $\geq 45$ years of age), never on any active osteoporosis therapy within the past 6 months and just been prescribed once weekly alendronate (Fosamax ${ }^{\circledR}$, Merck Sharp \& Dohme Corp., Pavia, Italy) or risedronate (Actonel ${ }^{\circledR}$ OSG Norwich Pharmaceuticals, Inc., North Norwich, NY, USA). BTMs are markedly reduced from baseline to 3 and 6 months later regardless of the type of bisphosphonates used. As both alendronate and risedronate were the most common bisphosphonates prescribed in the UMMC (23), patients who were either on alendronate or risedronate were included.

Exclusion criteria. Patients with metabolic bone disease or other medical conditions or treatment likely to affect bone metabolism, history of chronic renal, hepatic or gastrointestinal disease or traumatic lumbar compression fracture.

\section{Sample size}

To detect a mean difference of $5 \%$ with a pooled standard deviation of $10 \%$ between the control 
and intervention group, with $80 \%$ power of detection and $\alpha=0.05$, a sample size of at least 64 was required in each group (24). Assuming a $20 \%$ loss to follow up (15), the total number of participants required was at least 77 in each arm.

\section{Primary outcomes}

Primary outcomes measured were: medication adherence, BTMs and persistence. Adherence to bisphosphonate therapy is defined as the average percentage of participants who were both persistent (continued bisphosphonate therapy) and compliant (took medication in the correct manner on the scheduled day) (25).

Research in assessing medication adherence has been limited by the lack of a true gold standard (26). Three methods were used to assess medication adherence: direct-reporting (by asking the participant, 'How many doses of the alendronate/risedronate did you miss since the last time we met?'), pill count (by counting the number of bisphosphonate tablets left at each visit) and selfrecording (by asking participants to record the date they took their bisphosphonates). Persistence (defined as the time in days from the date of the first dose of bisphosphonate until discontinuation of treatment) was obtained from supply records using the pharmacy information system v8.6 (Ascribe, Bolton, UK).

Two BTMs: serum C-terminal cross-linking telopeptide of type I collagen (CTX-I), a bone resorption marker and serum osteocalcin (OC), a bone formation marker, were assessed so that the results could be compared and validated. Samples were collected using standard sampling tubes in the fasting state (between 8.00 and $9.00 \mathrm{AM}$ ) to minimize the unpredictable effects of feeding (27) and measured using electrochemiluminescence immunoassay (ECLIA) [Elecsys 1010® (serial no: 9003247); Roche Diagnostics, Mannheim, Germany]. Intra-assay variation was $0.96-1.91 \%$ and $1 \cdot 32-2 \cdot 61 \%$ for CTX-I $(n=30)$ and OC $(n=30)$ respectively. Inter-assay variation was $1 \cdot 81-2 \cdot 50 \%$ and $5 \cdot 30-6 \cdot 81 \%$ for CTX-I and OC respectively. Six tests were performed for each BTM over a 10-day period. Baseline and follow-up BTMs were run in the same assay to minimize inter-assay variation.

\section{PROCEDURE}

Potential participants were referred to the researcher by doctors at the osteoporosis, orthopaedic and menopause clinics in the UMMC. All participants gave their written informed consent and were provided with a patient information sheet.

This study used a stratified block randomization design to ensure that the number of participants on alendronate and risedronate in the control and intervention group were the same. Therefore, participants were first divided into whether they were on alendronate or risedronate, then randomly allocated to the intervention group using the random digits table (28) while the rest were allocated to the control group.

Participants were followed-up over a 12-month period which involved four visits (Fig. 1). All participants were dispensed 3 months' supply of bisphosphonate and instructed on how to take their medications. In addition, intervention participants received a 'counselling package' which consisted of an explanation on osteoporosis, risk factors, lifestyle modifications, goals of osteoporosis therapy, side effects and the importance of medication adherence. Verbal counselling was reinforced with an osteoporosis booklet. The pharmacist also reviewed participant's medications and conducted monthly follow-up via telephone calls for the first 6 months, then every 3 months until month 12. Control participants received no counselling.

All participants were requested to record the date they took their bisphosphonates and to bring back all remaining medications in their next visit for a pill count. An independent research assistant collected the data on medication adherence to minimize pharmacist interaction with control participants and to reduce intervention bias (29).

To ensure that study participants were a homogenous cohort with osteoporosis attributed to menopause, the renal function, full blood count, thyroid function, calcium, phosphate, alkaline phosphatase levels, intact parathyroid hormone (iPTH) and serum 25-hydroxyvitamin D [25(OH)D] were determined using standard automated clinical laboratory methods in the Central Diagnostic Laboratory, UMMC (ISO9001:2000 accredited). 


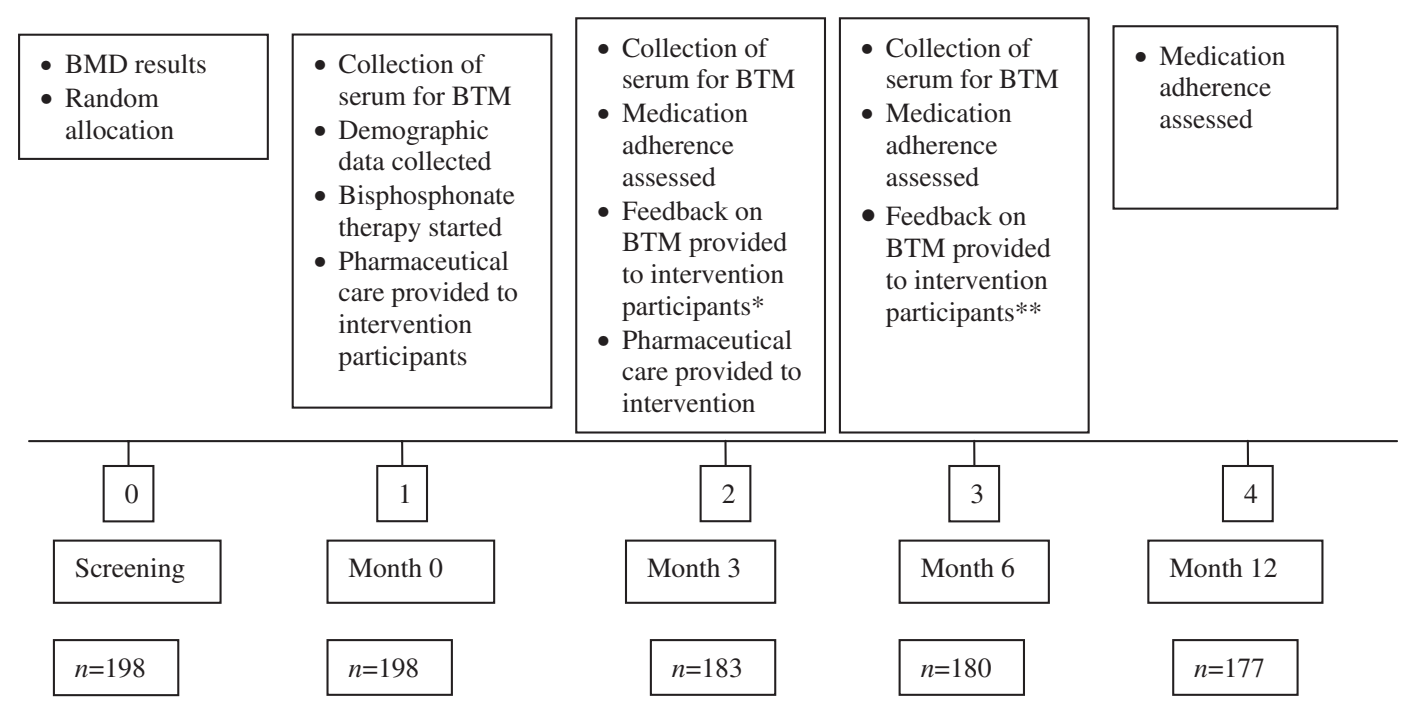

BMD=bone mineral density, BTM=bone turnover marker; * At month 4 , ** At month 7

Fig. 1. Time line of the study. BMD, bone mineral density; BTM, bone turnover marker; *at month 4 ; ${ }^{* *}$ at month 7 .

\section{Data analyses}

All data were entered into the SPSS version 15 (SPSS, Chicago, IL, USA). The impact of pharmaceutical care on medication adherence was analyzed using non-parametric Mann-Whitney $U$-test as normality assumptions could not be fulfilled. Persistence was analyzed using the Kaplan-Meier survival curve. The effect of intervention on persistence was analyzed using an extension of the Cox-regression model which involved a robust covariance matrix to adjust for within-cluster correlations (30). Statistical comparisons were expressed in terms of discontinuation hazard ratios (HRs). To identify potential factors that could be associated with medication adherence, a multiple regression analysis was conducted.

Changes in BTMs were calculated as differences between baseline, and at 3 and 6 months, expressed as a percentage of the median of all measurements. Participants who obtained at least a $55 \%$ or $40 \%$ decrease in CTX-I and OC, respectively, were considered to have a good BTM response, whereas participants who showed less than this least significant change were considered as nonresponders (31). The ability of a BTM to identify non-responders to bisphosphonate was evaluated using the McNemar test.

\section{RESULTS AND DISCUSSION}

One hundred and ninety-eight patients were recruited: intervention $=100$ and control $=98$ (Fig. 2). There was no difference in baseline characteristics between the control and intervention group (Table 1). The ethnic ratio of participants was similar to that of patients attending the osteoporosis clinic in the UMMC (32).

\section{Medication adherence}

In the present study, when medication adherence was assessed by direct-reporting, no significant difference was found between the control and intervention group (Table 2). This method of assessing medication adherence is easy to perform but is limited by recall bias of the participants. Direct-reporting only estimated the number of doses the patient missed, but did not account for how missed doses were adjusted.

When adherence was assessed by pill count, the intervention group showed a significantly higher adherence at month $6(P=0 \cdot 028)$. However, the pill count method only revealed whether the patient took her medication within that week or month, and not on which particular day. 


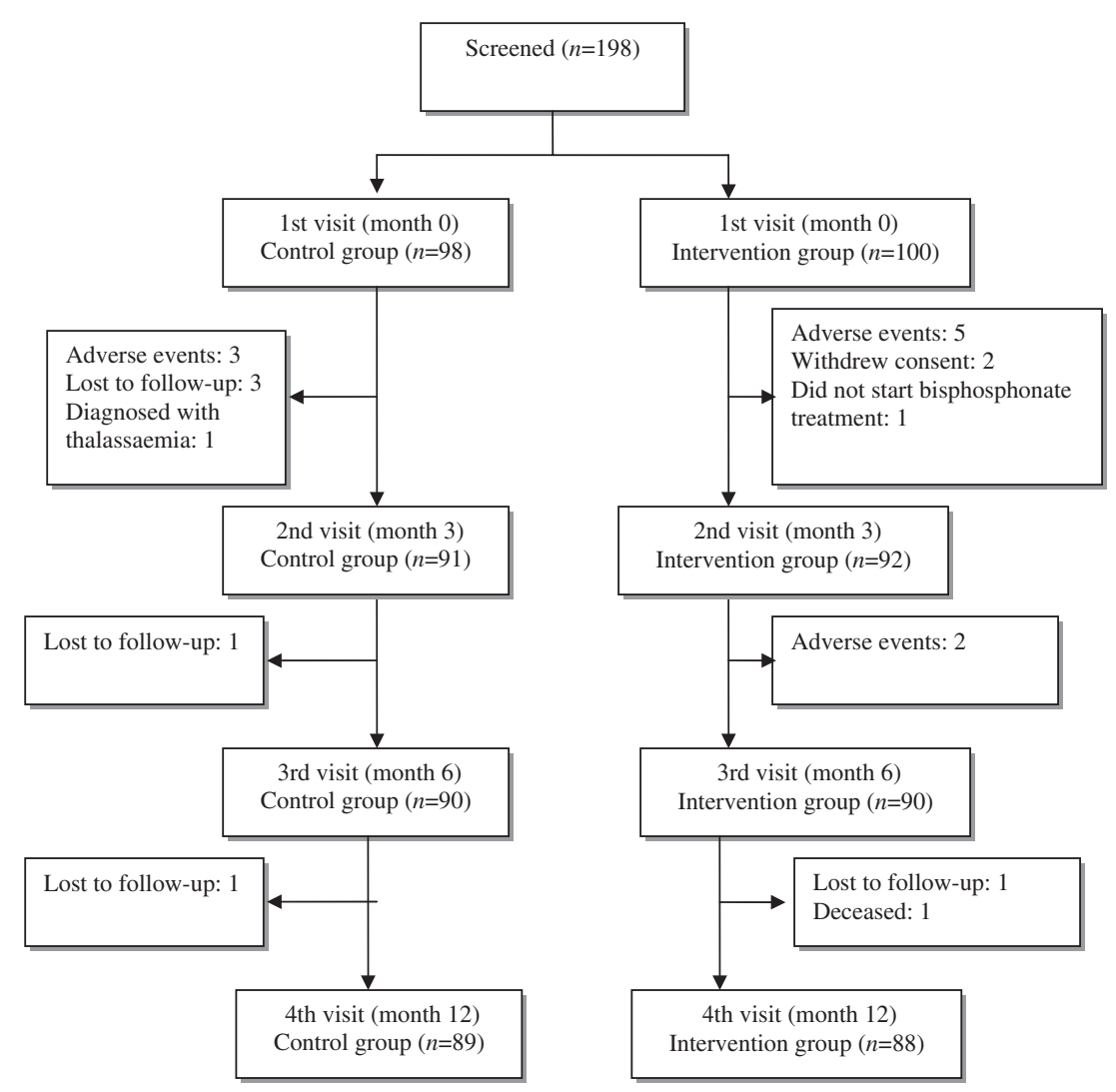

Fig. 2. Summary of the number of participants in the study.

When adherence was assessed using selfrecording by the participants, adherence at month 6 and 12 was also significantly higher in the intervention group compared with the control group ( $P=0.015$ and $P=0.047$ respectively). A high correlation was found between the self-recording and pill count method $(r=0.902-0.935, \quad P<0.001)$ which is similar to findings by Garber et al. (26) Therefore, results obtained from the self-recording method were used for subsequent analyses since the exact day when the participant took her weekly medication could be determined.

Absolute adherence to weekly bisphosphonate therapy is defined as the number of participants who took their medications exactly on the same day each week. As no significant difference in absolute adherence was detected between the control and intervention group, they were combined for subsequent analysis. Only 99 participants $(50 \%)$ took their weekly bisphosphonates exactly as instructed on the same day each week. However, due to the unique properties of the bisphosphonates, if the participant forgot to take her dose on the designated day of the week and took her missed dose the next day then returned to taking her dose on the scheduled day of the week again, she was still considered as adherent (33). This occurred in $38(38.8 \%)$ participants in the control group $[95 \%$ $\mathrm{CI}=29 \cdot 1-48 \cdot 4]$ and $38(38 \%)$ participants in the intervention group [95\% CI $=28 \cdot 5-47 \cdot 5]$. Participants in both the control and intervention group changed the days on which they took their medications by up to 5 days (10.1\%), and some even took it on different days each week (3.0\%).

Weekly execution of the dosing regimen among participants in the control and intervention group was very similar. Non-adherence to bisphosphonates included taking less than the prescribed amount $(43.4 \%)$ and not taking the medication on the scheduled day of the week $(76.8 \%)$. The most common reasons cited for non-adherence were: forgetfulness (75 participants), busy (33), away from home (28), avoiding side effects (6) and too many pills (5). This included participants who missed their doses but remembered to take them the following day. 
Table 1. Baseline characteristics of participants in the intervention study

\begin{tabular}{|c|c|c|c|c|}
\hline Characteristics & Control $(n=98)$ & $\begin{array}{l}\text { Intervention } \\
(n=100)\end{array}$ & ${ }^{\mathrm{b}} t$-value $/ \chi^{2}$ & $P$-value \\
\hline Mean age \pm SD (years) [range] & $67 \cdot 10 \pm 9 \cdot 55[42-94]$ & $65 \cdot 13 \pm 8 \cdot 98[44-86]$ & $1 \cdot 497$ & $0 \cdot 136$ \\
\hline \multicolumn{5}{|l|}{ Ethnicity $[n(\%)]$} \\
\hline Malay & $17(17 \cdot 3)$ & $14(14 \cdot 0)$ & \multirow[t]{3}{*}{$2 \cdot 642$} & \multirow[t]{3}{*}{$0 \cdot 450$} \\
\hline Chinese & $59(60 \cdot 3)$ & $55(55 \cdot 0)$ & & \\
\hline Indian and others & $22(22 \cdot 4)$ & $31(31 \cdot 0)^{\mathrm{a}}$ & & \\
\hline Mean BMI \pm SD & $23 \cdot 59 \pm 4 \cdot 20$ & $24 \cdot 04 \pm 4 \cdot 57$ & $-0 \cdot 718$ & $0 \cdot 474$ \\
\hline \multicolumn{5}{|l|}{ BMI range $[n(\%)]$} \\
\hline$<18.5$ (underweight) & $9(9 \cdot 2)$ & $11(11 \cdot 0)$ & \multirow[t]{4}{*}{$1 \cdot 464$} & \multirow[t]{4}{*}{$0 \cdot 691$} \\
\hline $18 \cdot 5-24 \cdot 9$ (normal) & $57(58 \cdot 2)$ & $50(50 \cdot 0)$ & & \\
\hline 25.0-29.9 (overweight) & $25(25 \cdot 5)$ & $29(29 \cdot 0)$ & & \\
\hline$\geq 30$ (obese) & $7(7 \cdot 1)$ & $10(10 \cdot 0)$ & & \\
\hline \multicolumn{5}{|l|}{ Level of education $[n(\%)]$} \\
\hline No formal education & $10(10 \cdot 2)$ & $6(6 \cdot 0)$ & \multirow[t]{4}{*}{$2 \cdot 616$} & \multirow[t]{4}{*}{$0 \cdot 455$} \\
\hline Primary education & $24(24 \cdot 5)$ & $19(19 \cdot 0)$ & & \\
\hline Secondary education & $39(39 \cdot 8)$ & $43(43 \cdot 0)$ & & \\
\hline Diploma/tertiary/postgraduate & $25(25 \cdot 5)$ & $32(32 \cdot 0)$ & & \\
\hline Had a previous fall or fracture $[n(\%)]$ & $50(51 \cdot 0)$ & $51(51 \cdot 0)$ & $1 \cdot 031$ & $0 \cdot 597$ \\
\hline No. years menopausal [mean \pm SD] & $18 \cdot 50 \pm 9 \cdot 75$ & $16 \cdot 28 \pm 9 \cdot 16$ & $1 \cdot 645$ & $0 \cdot 102$ \\
\hline Family history of osteoporosis $[n(\%)]$ & $15(15 \cdot 3)$ & $25(25 \cdot 0)$ & $3 \cdot 392$ & $0 \cdot 183$ \\
\hline \multicolumn{5}{|l|}{ Alcohol consumption $[n(\%)]$} \\
\hline Occasionally & $11(11 \cdot 2)$ & $14(14 \cdot 0)$ & \multirow[t]{2}{*}{$0 \cdot 346$} & \multirow[t]{2}{*}{$0 \cdot 557$} \\
\hline Never & $87(88 \cdot 8)$ & $86(86 \cdot 0)$ & & \\
\hline \multicolumn{5}{|l|}{ Smoking status $[n(\%)]$} \\
\hline Current smoker & $2(2 \cdot 0)$ & $1(1 \cdot 0)$ & \multirow{3}{*}{$0 \cdot 730$} & \multirow{3}{*}{$0 \cdot 694$} \\
\hline Ex smoker & $2(2 \cdot 0)$ & $1(1 \cdot 0)$ & & \\
\hline Never smoked & $94(95 \cdot 9)$ & $98(98 \cdot 0)$ & & \\
\hline \multicolumn{5}{|l|}{ Frequency of exercise $[n(\%)]$} \\
\hline$<3$ times/week & $50(51 \cdot 0)$ & $46(46 \cdot 0)$ & \multirow[t]{2}{*}{$0 \cdot 480$} & \multirow[t]{2}{*}{$0 \cdot 286$} \\
\hline$\geq 3$ times/week & $48(49 \cdot 0)$ & $54(54 \cdot 0)$ & & \\
\hline
\end{tabular}

aOne participant who was classified under 'others' was included under the Indian group.

${ }^{\mathrm{b}}$ The chi-square test was used for all categorical variables whilst the independent $t$-test was used for all continuous variables.

\section{Persistence}

Overall, persistence at 1 year was high and was similar between the control and intervention group. Using the log-minus-log plot, the hazards for the discontinuation of therapy were proportional throughout the study period, and the Kaplan-Meier survival curve showed no significant difference in persistence between the control and intervention group [log rank (Mantel-Cox) $\left.\chi^{2}=0.496, P=0.481\right]$. The proportion of participants who were persistent with bisphosphonate therapy after 12 months was $89 \cdot 8 \%$ and $87 \cdot 0 \%$ in the control and intervention group respectively. Those without back pain and those who consumed alcohol were more likely to drop out from therapy [hazard ratio (HR) $1.53 ; 95 \% \mathrm{CI}=$ $1 \cdot 10-2 \cdot 15, P=0 \cdot 013$ and $\mathrm{HR} 0.54 ; 95 \% \mathrm{CI}=0 \cdot 35-$ $0.85, P=0.008$ respectively]. Nine participants $(4.5 \%)$ discontinued their bisphosphonates within the first 3 months of therapy due to adverse drug effects. These include upper GI adverse effects (five participants), severe muscle or leg cramp (three participants) and lethargy (one participant).

\section{Bone turnover markers}

Both serum CTX-I and OC showed a rapid decrease within the first 3 months of therapy. 
Table 2. Adherence to once weekly bisphosphonates

\begin{tabular}{|c|c|c|c|c|c|c|c|c|}
\hline & \multirow[b]{2}{*}{ Month } & \multicolumn{2}{|l|}{ Control } & \multicolumn{2}{|l|}{ Intervention } & \multicolumn{3}{|c|}{ Mann-Whitney $U$-test } \\
\hline & & Mean \pm SD & Median & Mean \pm SD & Median & Mean rank & $z$-value & $P$-value \\
\hline \multicolumn{9}{|l|}{ Direct-reporting method } \\
\hline \multirow{3}{*}{$\begin{array}{l}\text { No. alendronate/risedronate } \\
\text { missed since the last visit }\end{array}$} & 3 & $94 \cdot 96 \pm 7 \cdot 01$ & $100 \cdot 00$ & $93 \cdot 21 \pm 10 \cdot 26$ & $100 \cdot 00$ & $95 \cdot 1888 \cdot 86$ & $-0 \cdot 898$ & $0 \cdot 369$ \\
\hline & 6 & $93.98 \pm 9.92$ & $100 \cdot 00$ & $93 \cdot 24 \pm 9 \cdot 17$ & $100 \cdot 00$ & $93 \cdot 8287 \cdot 18$ & -0.949 & 0.343 \\
\hline & 12 & $94 \cdot 27 \pm 12 \cdot 27$ & $97 \cdot 92$ & $94 \cdot 73 \pm 6 \cdot 56$ & $95 \cdot 83$ & $92 \cdot 4583 \cdot 49$ & $-1 \cdot 238$ & $0 \cdot 216$ \\
\hline \multicolumn{9}{|l|}{ Pill count method } \\
\hline & 3 & $97 \cdot 30 \pm 5 \cdot 35$ & $100 \cdot 00$ & $96 \cdot 57 \pm 7 \cdot 23$ & $100 \cdot 00$ & $92 \cdot 8289 \cdot 20$ & $-0 \cdot 602$ & 0.547 \\
\hline & 6 & $97 \cdot 01 \pm 7 \cdot 22$ & $100 \cdot 00$ & $98 \cdot 83 \pm 3.02$ & $100 \cdot 00$ & $82 \cdot 6494 \cdot 49$ & $-2 \cdot 191$ & $0 \cdot 028^{*}$ \\
\hline & 12 & $96 \cdot 46 \pm 10 \cdot 17$ & $100 \cdot 00$ & $97 \cdot 70 \pm 4 \cdot 68$ & $100 \cdot 00$ & $84 \cdot 3790 \cdot 78$ & -0.990 & $0 \cdot 322$ \\
\hline \multicolumn{9}{|l|}{ Self-recording method } \\
\hline & 3 & $97 \cdot 38 \pm 5 \cdot 33$ & $100 \cdot 00$ & $96 \cdot 85 \pm 6 \cdot 52$ & $100 \cdot 00$ & 93.0488 .98 & $-0 \cdot 683$ & $0 \cdot 495$ \\
\hline & 6 & $96.79 \pm 7.97$ & $100 \cdot 00$ & $98.91 \pm 3.23$ & $100 \cdot 00$ & $82 \cdot 2394.91$ & $-2 \cdot 426$ & $0 \cdot 015^{*}$ \\
\hline & 12 & $96 \cdot 17 \pm 10 \cdot 95$ & $100 \cdot 00$ & $97 \cdot 97 \pm 5 \cdot 25$ & $100 \cdot 00$ & $81 \cdot 7093 \cdot 57$ & -1.988 & $0 \cdot 047^{*}$ \\
\hline
\end{tabular}

*Statistically significant at $P<0.05$.

However, the change in serum OC was considerably slower compared with the change in serum CTX-I (Fig. 3a,b). There was no difference in serum CTX-I and serum OC reduction between the control and intervention group at months 3 and 6 . Only one non-responder to anti-resorptive therapy was identified at 6 months. There was significant difference between the CTX-I and OC in its ability to detect non-responders at 3 and 6 months [(McNemar $\left.\chi^{2}=33.03, P<0.001\right)$ and (McNemar $\chi^{2}=9 \cdot 31, P=0.003$ ) respectively].

The present study is one of the first prospective, randomized controlled trials (RCTs) conducted to assess the effects of pharmaceutical care on adherence (and its association with BTMs), as well as persistence with osteoporosis therapy. Intervention participants who received pharmaceutical care reported significantly higher medication adherence compared with the control group, indicating the benefits of providing pharmaceutical care. However, pharmacist intervention did not affect persistence to osteoporosis therapy at 1 year or the BTM of the participants, at up to 6 months.

Bisphosphonates required specific instruction on timing and how it should be taken (34). This emphasizes the importance of counselling patients on the proper method of taking bisphosphonates to improve adherence. Prompt actions can be taken if poor adherence or persistence is identified, which includes reassuring patients about the medication's side effects and how to prevent or alleviate the symptoms of adverse drug reactions and pain.

Due to the long effect of bisphosphonates on the bone (35), as long as participants take their missed dose of bisphosphonate within 2 days of the scheduled day, they can still be defined as adherent (33). Using this definition, the overall medication adherence was very good $(>95 \%)$. However, if adherence was defined as participants who only took their weekly dose exactly on the scheduled day of the week, adherence to weekly bisphosphonates was reduced to only $50 \%$, which is similar to other medication adherence studies with once-daily dosing (36-38). The two main reasons for non-adherence to bisphosphonate therapy were, taking less than was prescribed and changing the day they took their bisphosphonates (which resulted in a difference of up to 5 days). Nine participants $(4.5 \%)$ discontinued their bisphosphonates due to adverse drug reactions and pain attributed to the medication, which was similar to findings of previous studies (39-41).

The persistence rates with weekly bisphosphonates in both the control and intervention group were higher than expected $(89.8 \%$ and $87.0 \%$ respectively). Adherence and persistence tend to be higher in clinical trials than in real life, especially when participants in both the control and intervention group have intensive follow-up sessions (13). Patient motivation may also be higher than in 
(a) Changes in CTX-I in the control and intervention group

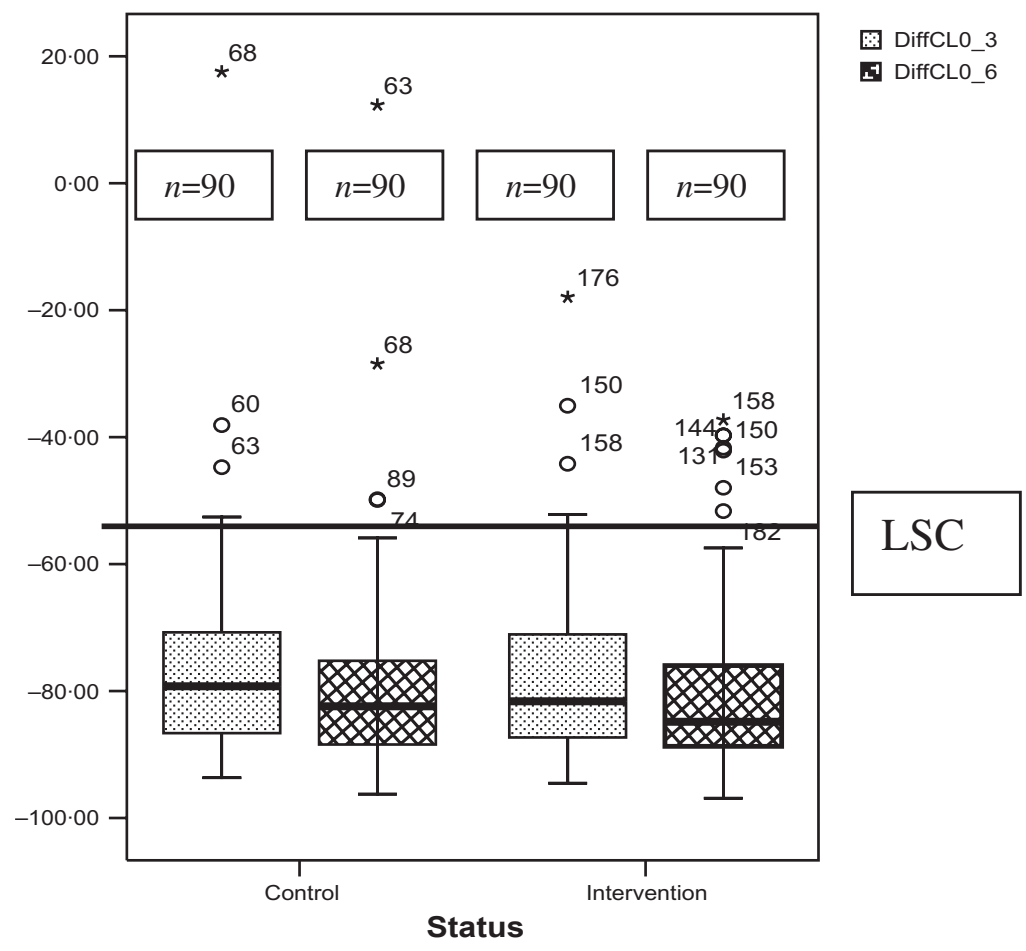

(b) Changes in $\mathrm{OC}$ in the control and intervention group

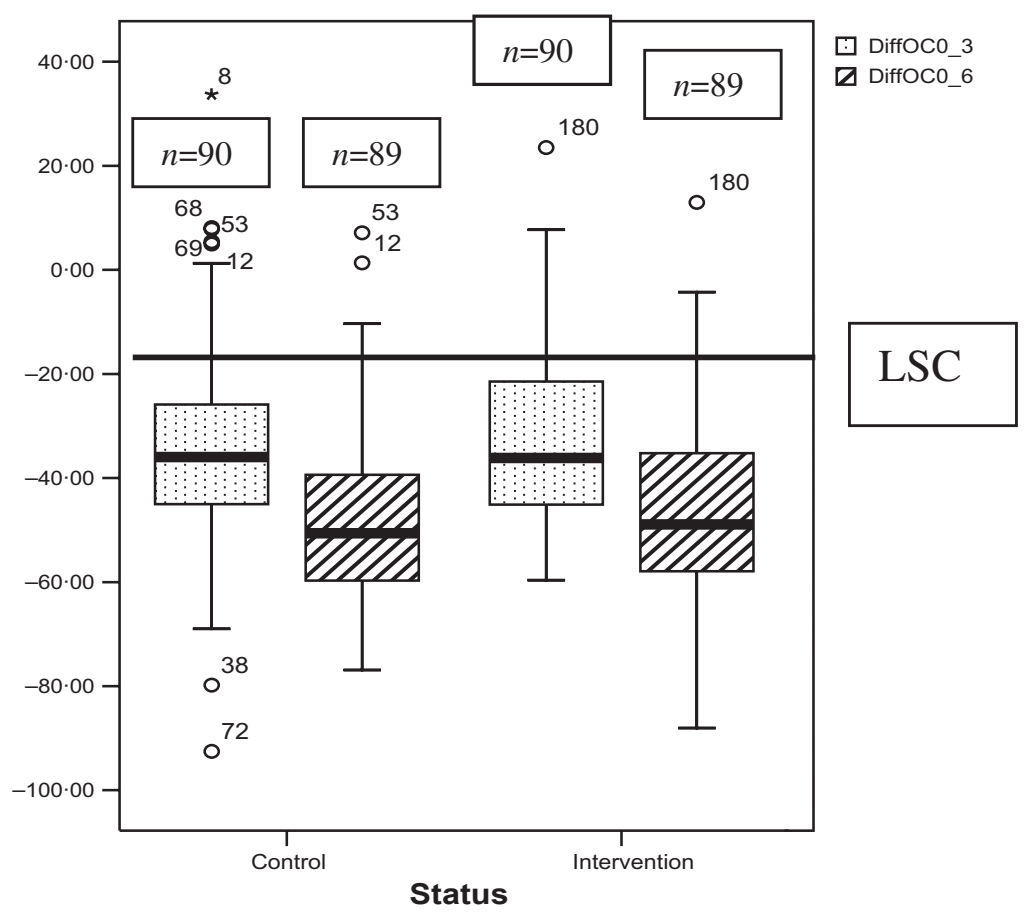

Fig. 3. (a) Changes in CTX-I in the control and intervention group. (b) Changes in OC in the control and intervention group. Results are expressed as the median percentage change from baseline at 3 and 6 months respectively. Using the Mann-Whitney $U$-test, no significant difference was detected between the control and intervention group for CTX-I at 3 and 6 months $(P=0.264$ and $P=0.372$ respectively) as well as for OC at 3 and 6 months $(P=0.709$ and $P=0.291$ respectively). CTX-I, serum concentration of the C-terminal telopeptide of collagen type I; OC, serum concentration of osteocalcin; LSC, least significant change. 
actual practice as participants were aware that the pharmacist was assessing their medication adherence and persistence as one of the primary outcomes in this study. The situation in real practice conditions may be different. Therefore, further observational studies should be conducted to supplement the data obtained from the present RCT in order to depict a more practice-based scenario.

It was difficult to compare the adherence and persistence rates between the present study and other RCTs (12-14), as each study defined and assessed medication adherence or persistence differently. In addition, different studies assessed adherence and persistence using different medications (once daily raloxifene/risedronate vs. once weekly alendronate/risedronate). The convenience of the weekly dosing probably accounts for the higher adherence and persistence rate in the present study as compared with previous studies $(12,13)$.

There was no significant difference between the BTMs of the control and intervention participants in this study at 3 and 6 months. The lack of significant correlation may be due to the already high medication adherence observed in both the control and intervention group and hence did not affect the BTMs. In addition, the half-life of bisphosphonates is very long (1.5-10 years) and its serum levels are only transiently elevated after each dose (42). As a result, if adherence is interrupted at weekly intervals, most of the active sites will be only a few days old on an average and relatively little resorption will occur and hence, this small change will not be detected by the BTMs. Changes in the BTMs will only be detected if the patient stops bisphosphonate therapy for more than a month (43).

Bone turnover markers monitoring by nurses improved medication adherence (12), while monitoring by physicians did not (13). The differences could be due to the medication studied (as discussed earlier) and the methodology used. When participants were informed of their poor BTM response, the rate of discontinuance was higher than those who were informed of a good BTM response (13). It was not possible to determine this outcome in the present study as only one participant had a poor BTM response and was identified as a non-responder. However, the major advantage of using a BTM is that by 3 months, non-responders to therapy can be detected. This is considerably shorter than the 2 years required for a reliable BMD test.

The major strength of this study is that it assessed the effectiveness of pharmaceutical care on medication adherence in postmenopausal osteoporotic women. It also attempted to determine any possible association between medication adherence and objective measures such as the use of BTMs. However, one of the limitations in this study is that data were collected from only one site and hence cannot be considered as population-based.

\section{WHAT IS NEW AND CONCLUSIONS}

The provision of pharmaceutical care improved medication adherence but not persistence. BTMs were not appropriate objective measures for assessing adherence to weekly bisphosphonates but were useful for identifying non-responders to treatment within 3-6 months, much earlier than using BMD. The study indicates that pharmacists have a role in improving medication adherence, but its long-term effect on persistence warrants further studies with longer duration.

\section{ACKNOWLEDGEMENTS}

We thank Professor Dr Awang Bulgiba (Department of Social and Preventive Medicine, University of Malaya) and Professor Dr Kulentheran Arumugam (Department of Obstetrics and Gynaecology, University of Malaya) for their advice on statistical procedures. We would also like to express our appreciation to the Central Diagnostic Laboratory of the UMMC and its staff for allowing us to conduct all the BTM tests in the laboratory and for their assistance and cooperation. Last but not least, our greatest appreciation to all the participants of this study who had kindly spared their time to make this study a success.

\section{REFERENCES}

1. Cranney A, Guyatt G, Griffith L (2002) IX Summary of meta analyses of therapies for postmenopausal osteoporosis. Endocrine Reviews, 23, 570-578.

2. Pols HAP, Felsenberg D, Hanley DA et al. (1999) Multinational, placebo-controlled, randomized trial of the effects of alendronate on bone density and fracture risk in postmenopausal women with low 
bone mass: results of the FOSIT study. Osteoporosis International, 9, 461-468.

3. Harris ST, Watts N, Genant HK et al. (1999) Effects of risedronate treatment on vertebral and nonvertebral fractures in women with postmenopausal osteoporosis. JAMA, 282, 1344-1352.

4. Weycker D, Macarios D, Edelsberg J, Oster G (2006) Compliance with drug therapy for postmenopausal osteoporosis. Osteoporosis International, 17, 16451652.

5. Lappe J (2006) Nonadherence to osteoporosis medications: a critical problem. Clinical Reviews in Bone and Mineral Metabolism, 4, 25-32.

6. Siris E, Harris S, Rosen C et al. (2006) Adherence to bisphosphonate therapy and fracture rates in osteoporotic women: relationship to vertebral and nonvertebral fractures from 2 US claims databases. Mayo Clinic Proceedings, 81, 1013-1022.

7. Sebaldt RJ, Shane LG, Pham B, Cook R, Thabane L, Petrie A (2004) Long-term effectiveness outcomes of non-compliance and non-persistence with daily regimen bisphoshonate therapy in patients with osteoporosis treated in tertiary specialist care. Osteoporosis International, 15, [Abstract P391SA].

8. Tosteston AN, Grove MR, Hammond CS et al. (2003) Early discontinuation of treatment for osteoporosis. American Journal of Medicine, 15, 209-216.

9. Eastell R, Garnero P, Vrijens L, Van de Langerijt L, Pols HAP, Ringe JD (2003) Influence of patient compliance with risedronate therapy on bone turnover marker and bone mineral density response: the IMPACT study. Calcified Tissue International, 72, [Abstract P297].

10. Nielsen D, Ryg J, Nissen N, Nielsen W, Knold B, Brixen K (2008) Multidisciplinary patient education in groups increases knowledge on osteoporosis: a randomized controlled trial. Scandinavian Journal of Public Health, 36, 346-352.

11. Lai P, Chua SS, Chan SP (2010) A systematic review of interventions by healthcare professionals on community-dwelling postmenopausal women with osteoporosis. Osteoporosis International, doi 10.1007/ s00198-010-1199-0.

12. Clowes JA, Peel NFA, Eastell R (2004) The impact of monitoring on adherence and persistence with antiresorptive treatment for postmenopausal osteoporosis: a randomized controlled trial. Journal of Clinical Endocrinology and Metabolism, 89, 1117-1123.

13. Delmas PD, Vrijens B, Eastell R et al. (2007) Effect of monitoring bone turnover markers on persistence with risedronate treatment of postmenopausal osteoporosis. Journal of Clinical Endrocrinology and Metabolism, 92, 1296-1304.
14. Guilera M, Fuentes M, Grifols M, Ferrer J, Badia X (2006) Does an educational leaflet improve selfreported adherence to therapy in osteoporosis? The OPTIMA study. Osteoporosis International, 17, 664-671.

15. Schousboe JT, DeBold RC, Kuno LS, Weiss TW, Chen YT, Abbott TA (2005) Education and phone followup in postmenopausal women at risk for osteoporosis - effects on calcium intake, exercise frequency, and medication use. Disease Management and Health Outcomes, 13, 395-404.

16. Hepler CD, Strand LM (1990) Opportunities and responsibilities in pharmaceutical care. American Journal of Hospital Pharmacy, 47, 533-543.

17. Jaglal SB, Hawker G, Bansod V et al. (2009) A demonstration project of a multi-component educational intervention to improve integrated post-fracture osteoporosis care in five rural communities in Ontario, Canada. Osteoporosis International, 20, 265-274.

18. MacLaughlin EJ, MacLaughlin AA, Snella KA, Winston TS, Fike DS, Raehl CR (2005) Osteoporosis screening and education in community pharmacies using a team approach. Pharmacotherapy, 25, 379-386.

19. Keely JL (2002) Pharmacist scope of practice. Annals of Int Medicine, 136, 79-85.

20. Delmas PD (2000b) Markers of bone turnover for monitoring treatment of osteoporosis with antiresorptive drugs. Osteoporosis International, 11(Suppl. 6), S66-S76.

21. Reginster JY, Henrotin Y, Christiansen C (2001) Bone resorption in post menopausal women with normal and low BMD assessed with biochemical markers specific for telepeptide derived degradation products of collagen type 1. Calcified Tissue International, 69, 130-137.

22. Delmas PD (2004) Advances in osteoporosis research using biochemical markers of bone turnover. 22nd Annual meeting of the American Society for Bone and Mineral Research, 22-24 (abstract).

23. Pharmacy Information System (2005) Data on file from the University Malaya Medical Centre, using Ascribe version 8.6. Bolton: Pharmacy Information System.

24. Dupont WD, Plummer WD (2004) PS: power and sample size calculation. Available at: http://biostat. mc.vanderbilt.edu/twiki/bin/view/Main/Power SampleSize. (accessed 30 June 2007).

25. International Osteoporosis Foundation (2006) Staying power: closing the adherence gap in osteoporosis. Available at: http://www.iofbonehealth.org/down load/osteofound / filemanager/publications / pdf / closing-adherence-gap.pdf. (accessed 22 August 2008).

26. Garber MC, Nau DP, Erickson SR, Aikens JE, Lawrence JB (2004) The concordance of self-report with 
other measures of medication adherence: a summary of the literature. Medical Care, 42, 649-652.

27. Clowes JA, Hannon RA, Yap TS, Hoyle NR, Blumsohn A, Eastell R (2002) Effect of feeding on bone turnover markers and its impact on biological variability of measurements. Bone, 30, 886890.

28. Abramson JH, Abramson ZH (1999) Survey methods in community medicine, 5th edn. Edinburgh: Churchill Livingstone.

29. Roughead EE, Semple SJ, Vitry AI (2005) Pharmaceutical care services: a systematic review of published studies, 1990 to 2003, examining effectiveness in improving patient outcomes. International Journal of Pharmacy and Practice, 13, 53-70.

30. Donner A, Klar N (2000) Design and analysis of cluster randomization trials in health design. London: John Wiley \& Sons, 128-138.

31. Delmas PD, Eastell R, Garnero P (2000) The use of biochemical markers of bone turnover in osteoporosis. Osteoporosis International, 11, S2-S17.

32. UMMC Medical Records Department (2005) Data on file: number of osteoporosis patients who attended the outpatient clinics in the University Malaya Medical Center. Kuala Lumpur: Medical Records Department of the University Malaya Medical Center.

33. Fosamax. (1999) Product Information (alendronic acid). Whitehouse Station: Merck \& Co., Inc.

34. Downey T, Foltz S, Boccuzzi S, Omar M, Kahler K (2006) Adherence and persistence associated with the pharmacologic treatment of osteoporosis in a managed care setting. Southern Medicine Journal, 99, 570-575.
35. Schnitzer T, Bone HG, Crepaldi G et al. (2000) Therapeutic equivalence of alendronate $70 \mathrm{mg}$ once weekly and alendronate $10 \mathrm{mg}$ daily in the treatment of osteoporosis. Aging (Milano), 12, 1-12.

36. McDonald HP, Garg AX, Haynes RB (2002) Interventions to enhance patient adherence to medication prescriptions. JAMA, 288, 2868-2879.

37. Clifford RM, Davis WA, Batty KT, Davis T (2005) Effect of a pharmaceutical care program on vascular risk factors in type 2 diabetes: The Fremantle Diabetes Study. Diabetes Care, 28, 771-776.

38. Haynes RB, McDonald HP, Garg AX (2002) Helping patients follow prescribed treatment - clinical applications. JAMA, 288, 2880-2883.

39. Rizzoli R (2007) What factors determine patient adherence to osteoporosis treatment regimens? Nature Clinical Practice. Endocrinology Metabolism, 3, 8081.

40. Papaioannou A, Kennedy C, Dolovich L, Lau E, Adachi J (2007) Patient adherence to osteoporosis medications: problems, consequences and management strategies [Review]. Drugs and Aging, 24, 37-55.

41. Solomon D, Avorn J, Katz J et al. (2005) Compliance with osteoporosis medications. Archives of Internal Medicine, 15, 2414-2419.

42. Watts N, Freedholm D, Daifotis A (1999) The clinical tolerability of alendronate. International Journal of Clinical Practice, 101, 51-61.

43. Tanko LB, Mouritzen U, Lehman HJ et al. (2003) Oral ibandronate: changes in markers of bone turnover during adequately dosed continuous and weekly therapy and during different suboptimally dosed treatment regimens. Bone, 32, 687-693. 